\title{
Describing Life: Towards the Conception of Howard Pattee
}

\author{
Lukáš Hadwiger Zámečník, Jaroslav Krbec \\ Palacký University, Department of General Linguistics, Olomouc, Czech Republic
}

Received 25 November, 2018; Accepted 25 April, 2019

\begin{abstract}
A description of living systems is still a topic of discussion among a number of disciplines. By an evaluation of the approaches, we get to an axis differentiating those that are indisputable in sense of dealing with verifiable and measurable phenomena. We thus also get to approaches that integrate particular extensions when dealing with the possibilities to describe living systems and processes. It is a task for biosemiotics to find connections of these approaches and thus ways to enrich each other or simply describe phenomena to the widest extent possible. One of the authors whose work is permeated by this idea is Howard Pattee. Inspired by his work, we discuss the options of description when talking about living systems and semiotic apparatuses. We do so by a formulation of two viewpoints that differ in questions of contextual dependency, interpretation and necessity of the existence of an autonomous agent as indispensable elements for the description of life phenomena.
\end{abstract}

Keywords: code $\bullet$ context $\bullet$ interpretation $\bullet$ self-replication $\bullet$ von Neumann probe

(C) Sciendo

\section{Quotation}

“Ce qui est premier, ce n'est pas le commencement, mais le recommencement, et l'être, c'est précisément l'impossibilité d'être une première fois.

Mouvement qu'on peut éclairer - non expliquer - en évoquant ces formes et ces crises appelées « complexes ». Leur essence, c'est qu'au moment où elles se produisent, elles se sont déjà produites, elles ne font jamais que se reproduire; c'est là leur trait caractéristique: elles sont l'expérience du recommencement. « A nouveau, à nouveau! », c'est le cri de l'angoisse aux prises avec l'irrémédiable, avec l'être. A nouveau, à nouveau, telle est la blessure fermée du complexe: cela a lieu à nouveau, cela recommence, encore une fois. Le recommencement de l'expérience, et non pas le fait que celle-ci ne réussit pas, voilà le fondement de l'échec. Tout recommence toujours - oui, encore une fois, à nouveau, à nouveau."

Blanchot, M. L'espace littéraire, 1955 Paris: Gallimard, pp. 255-256.

\section{Introduction}

In general, a semiotic dimension is the necessary supplement for describing living systems and the processes of related interactions. It is not only this descriptive extension but the functional potential of the semiotic control itself, which autonomous systems exert and which permits their existence in time. ${ }^{1}$ Inspired by the work of Howard Pattee, we discuss the tools for describing the semiotic dimension in relation to conditions for the very existence of living organisms.

1 Talking about the functional potential of the autonomous agent, we are aware that there are possibilities to distinguish metasemiotic and semiotic capabilities. As topics discussed further refer to various aspects of description, we consider this distinction to be evident in the context of individual subchapters.

\footnotetext{
*Corresponding author:E-mail: Iukas.zamecnik@upol.cz, jkrbec@email.cz 
By characterizing the semiotic apparatus available and asserting the necessity of particular elements intrinsic and extrinsic to a system, we gradually formulate two viewpoints differing in questions of contextual dependency, interpretation and necessity of the existence of an autonomous agent as an indispensable element when describing living systems. The dialogic nature of this paper also reflects the existence of various possible approaches within the life sciences.

In the first part, we posit the role of semiotic processes in the physical world. Based on the essential differentiation of constraints in various types of systems, we aim for a description of a semiotically fundamental agent category in the form of the functional potential of von Neumann probe.

In the second part, we do not refute these cited principles but rather expand them in the sense of extending necessary semiotic elements for describing and when talking of autonomous systems. We do this within the context of the involvement of constraints and by reflecting on concepts based on the assumed existence of two types of complementary modes. Fundamentally, these modes are then closely linked to the theoretical basis of von Neumann's system replication cited in the first part but with regard to various concepts of the epistemic cut.

\section{Between Semiotic and Physical Domains}

We do not question the basic thesis that there is a mutual link between the physical domain and the domain of semiotics. ${ }^{2}$ The nature of this nexus constitutes the major area of our analysis. In the broad sweep of biosemiotic literature, it is Howard Pattee who has most insistently expressed the need for such an investigation, indeed claiming to perform biosemiotics while not neglecting physics:

"It is not clear to me what biosemiotics wants to be. All I can suggest is that if its practitioners want it to be accepted as science rather than philosophy, they must focus more on empirically decidable models, rather than emphasizing its linguistic and philosophical foundations. In other words, if biosemiotics claims that symbolic control is the distinguishing characteristic of life, and if it also claims to be a science, then it must clearly define symbols and codes in empirical scientific terms that are more familiar to physicists and molecular biologists". [Kull and Pattee, p. 328]

In the context of the semiotic conceptualization of biology, the problem of this relationship between the physical and living domains of investigating is astutely expressed by Kull: "(...) how the possibility of choosing one's path - a characteristic feature of all life - can be embedded into the picture of physical world which is based on inexorable physical laws" [1, p. 311]. As a central problem in the relationship under investigation, it seems to be primarily determinism associated with the physical domain and specifically with the laws of physics. Probably, Pattee's greatest contribution is to clarify this problem as a conceptual misunderstanding. Pattee has consistently distinguished between dependence on law and determination by law.

We cannot conceive living systems as the result of a process determined by physical laws - we cannot simply explain their presence by subsuming them under a general law. Pattee sees determinism as inadequate because the explanatory puissance of physical laws is strictly limited. Consequently, Pattee refers to the compatibility of a set of deterministic physical laws with a given set of results that cannot be decided on as a result of mere knowledge of physical laws. This finding, however, has another aspect. Pattee adduces "that lawfully indeterminate does not mean physically indistinguishable" [1, p. 313]. However, despite the inadequacy of determinism, there is not strict separation of the physical and living domains. That there is a system not determined by physical law does not mean that it is inaccessible to physical description and possibly explanation. Dependence on law expresses another type of relationship than a deterministic relationship.

Pattee then approaches the problem with another conceptual distinction between laws and constraints. These constraints can best be characterized as a set of heterogeneous conditions [2, p. 285] choosing which physically defined but by physical law ambiguously determined state of the system (outcome of the process) will actually be realized.

2 Note that our research is not based on the a priori determination of any methodological or even ontological position, e.g. reductionism, nor is it just a conceptual analysis that would map the space between physical and semiotic concepts. Our efforts aim at a consistent content analysis of the relationship between semiotics and physics on the one hand and semiotics and metamathematics on the other hand. 
Pattee's system highlights the fundamental problem in describing physical or living dynamic systems that pass phase transition. ${ }^{3}$ For Pattee and us, it is not a matter of finding various vague analogies or even metaphors. Pattee understands a described mechanism as a fundamental tool to clarify the bond between the living and the physical domains. In this context, Pattee introduces the concept "frozen accident": "Such symmetry-breaking events that persist for structural, functional, or selective reasons are appropriately called 'frozen accidents'” [1, p. 313]. We are aware that the concept frozen accident deserves a more detailed interpretation, but we use it here merely to adhere to the logos of the argument. ${ }^{4}$

According to Pattee, the freezing of a certain configuration of a physical system, conservation of various transitions of state, represents an essential bridge between the physical and living domains. The problem of the relationship between physical and living domains is thereby suspended, transformed by the conceptual apparatus of dynamic systems theory, whereby this theory uses the mechanism of spontaneous symmetry breaking. ${ }^{5}$

Pattee inspiringly deals with the problem of linking the physical and living domains. As previously indicated, dynamic systems theory is a set of tools marginalizing the traditional conceptual difficulties of the relationship between the domains of disciplines. However, the semiotic view of biology requires a detailed definition of the individual kinds of constraints because, as Kull points out, "Constraints exist in non-living, living and mental world, but they fundamentally differ" [1, p. 317].

Pattee is aware of this difficulty, and thus, in addition to defining a certain hierarchy of constraints [2, p. 285], he also offers a detailed definition of constraint(s) related to semiotic control of living systems. This will be outlined later in the context of Peircean tradition. Despite efforts to define the concept of constraint carefully, we reserve some critical remarks.

\section{Problems with Constraints}

First, the hierarchy of constraints varies, and this variation is not clearly standardized and unified only terminologically. Basically, in the context of the theory of dynamic systems, Pattee uses the general constraint characteristic as "(...) [constraints] enter the lower level dynamics as boundary conditions on the possible motions" [2, p. 285]. This definition, understandable on the theoretical plane for a dynamic system, is not clearly tangible when we speak of the whole hierarchy: "(...) from the chemical bonds that constrain gene sequences, enzyme dynamics, and cell membranes, to the matter-symbol transition of the structural genes and the epigenetic controls of development, and finally to the architecture of the nervous system and the brain" [1, p. 316].

What is important is that the vast majority of constraints, although significant in terms of the link between various domains, are irrelevant to the semiotic interpretation of biology. More precisely, constraints in the fields of physics and chemistry are irrelevant because they do not refine the problem of the differentiation between the physical system and the semiotic system (because they still create non-semiotic systems). Consistently, the spontaneous symmetry breaking is thus irrelevant, as it optimally only distinguishes domains but does not express the specificity of the domains.

Nevertheless, the constraints of the upper level domains (neuroscience, psychology, etc.), operating with concepts such as an individual, an interpreter and memory, are also irrelevant. Mostly, it is only as a metaphor, but primarily because these constraints are complex entities, themselves needing explanation (such as in terms of the already mentioned concepts of an individual, an interpreter - a subject of semiosis - and memory).

Therefore, we concede that the simplest option of selecting constraints specifying the domain is not only metaphorical, representing the lower limit of a semiotic system. We also concede that such a field of semiotics is also sufficiently defined by metamathematical terms, more precisely through the concepts of the theory of proofs (Gödel), the theory of algorithms (Turing) and the theory of self-reproducing automata (von Neumann).

3 This concept and the concepts associated with are interdisciplinary applicable. Pattee uses the physical terminology directly: "Since all the basic laws of physics are expressed in terms of energy, systems with two or more states with the same energy are lawfully indeterminate. However, we can often distinguish these states by measuring their initial conditions. These law-equivalent states are often called degeneracies or symmetries". (P1], p. 313)

4 For more information, see [21]P primarily chapters: Laws and Constraints, Symbols and Languages (pp. 81-90), The Physical Basis and Origin of Hierarchical Control (pp. 91-110) and Clues from Molecular Symbol Systems (pp. 155-164).

5 For contemporary discussion concerning phase transitions and spontaneous symmetry breaking, see Batterman, [3], primarily chapters of Leo Kadanoff, Tarun Menon and Craig Callender, Jonathan Bain, Robert Batterman and Sorin Bangu. 


\section{Von Neumann Probe as the Basic Semiotic System}

In the context of Pattee's definition of one constraint: "the matter-symbol transition of the structural genes" [1, $p$. 316], as such, is a constraint related to the description/construction differentiation, the well-known von Neumann probe. This concept illustrates the basic condition of self-replication as a semiotic condition, in the sense that self-replication requires the existence of a symbolic record, the linearity of this record and the arbitrariness of the assignment of expression and content (so-called double sets condition) [4, p. 18].

There is little consensus concerning the interpretation of the meaning of von Neumann probe and the significance of self-replication for biology and semiotics, let alone for biosemiotics. ${ }^{6}$ Pattee again stresses the importance of von Neumann's theory of self-reproducing automata in the biosemiotic sphere, specifically in connection with the condition of the existence of a symbolic record. If, however, for Pattee, self-replication is the minimum logical condition of evolution [1, p. 286], then for Barbieri, for example, the mechanism of self-replication is not enough [5, pp. 174-175]. In the following, we assume that von Neumann probe can be considered to be a semiotic system. ${ }^{7}$

The indispensability of a symbolic record allowing representation of the constituent elements of the von Neumann's system has been expressed by himself with the current reference to logical paradoxes that otherwise enter the process of self-reproduction:

"In the passive method the self-reproducing automaton contains within itself a passive description of itself and reads this description in such a way that the description cannot interfere with the automaton's operations. In the active method the self-reproducing automaton examines [inspection] itself and thereby constructs a description of itself. Von Neumann suggests that this second method would probably lead to paradoxes of the Richard type, and for this reason he adopts the first method" [6, pp. 125-126].

Here, we see the alignment of the basic semiotic conditions, namely, the existence of a symbolic record/double sets condition, with the paradoxes of self-reference. In other words, self-reproduction requires a semiotic condition for the existence of a symbolic record, which itself is also a condition preventing the occurrence of paradoxes of self-reference. Reproduction is possible only through description, not through the inspection leading to paradoxes of self-reference. We hold that this linking of the basic semiotic conditions with logical/metamathematical conditions cannot be overlooked. On the contrary, we are convinced that exploring this relationship can shed new light on the nature of the simplest semiotic systems.

An interesting aspect of our research is the discovery that at the heart of the theory of self-reproduction lies the recognition of limits of the purely syntactic description of symbolic systems such as we learn from Gödel and Turing. The relationship between Gödel's findings in the field of theory of proofs and Turing's findings in the field of algorithm theory is well known. Less known is that von Neumann's theory of self-reproducing automata is also related to this pair.

In all three theories, the basic link is the condition of the existence of a "double set", respectively the existence of arbitrary but stable ${ }^{8}$ pairing between two sets. This condition is present in the concept of Gödel's numbering, which assigns to each element of the system under consideration (say to each logical/mathematical symbol), arbitrarily but steadily, a natural number. This numbering then allows, without the occurrence of paradoxes of self-reference, the arithmetization of metamathematics. For Turing, the numbering, and thus ordering, of individual Turing machines, enables the possibility of constructing a universal Turing machine. ${ }^{9}$ For von Neumann, the organized set of descriptions of the elements of the reproduced system allows for self-reproduction - the existence of von Neumann's probe.

The necessity of abandoning a purely syntactical approach to symbolic systems and introducing a distinction between syntax and semantics provides an interesting conceptual modulation, which again reflects in all three theories of proofs, algorithms and self-reproducing automata.

6 For specification, see Faltýnek and Jastřembská, 24], pp. 179-180, c. 12.

7 It is appropriate to point out that there are also aspects of materiality when discussing semiotic systems, however not when viewing semiotics as a part of metamathematics.

8 Here we see why Pattee uses the definition of constraint in connection with the concept of "rozen accident" e do not think that Pattee himself directly refers to von Neumann's, Gödel's and Turing's relationship.

9 For more details, see Hofstadter 235] and Partee et al. 17]. 
The best-known proof is Gödel's discovery of the need to conceptually differentiate provability (syntax) and truthfulness (semantics). A true sentence may be a non-provable sentence, although any provable sentence is true. What is provable is true, but what is true does not have to be provable.

In Turing's theory of algorithms, we have to distinguish between decidability (syntax) and acceptability (semantics) of a string. An acceptable string can be an undecidable string, although each decidable string is also an acceptable string. What is decidable is acceptable, but what is acceptable need not be decidable [7, pp. 520-523].

For von Neumann's theory, we append a reflective discovery related to the need to differentiate between constructability (syntax) and representability (semantics). The representable code can be an unconstruable code, although each construable code is necessarily a representable code. What is construable is also representable/ readable, but what is representable/readable need not be construable. ${ }^{10}$

Our discovery of the identical condition of double sets in theory of proofs, algorithms and self-reproducing automata posits an interesting dilemma, consisting of a two-way response to the following question and the implications of the answers:

Is the existence of a symbolic code - a double set of arbitrary but stable mutual relations - the minimum necessary and sufficient condition of the semiotic system?

In sum, is von Neumann probe a semiotic system?

If we affirm this, then we find a simple and coherent theory that lies at the foundation of semiotics. We need no concepts such as interpretation or subject of semiosis, and we find the frontier of the semiotic system. Moreover, important for biosemiotics: it is relevant to talk about the semioticity of biology, i.e. the biosemiotics acquires solid foundations, which, inter alia, Pattee also cites. On the other hand, semiotics is expressible/reducible to the basic level of metamathematics; von Neumann probe represents another isomorphic representation of the equal problem of distinction between syntax and semantics:

Could it be that all the semiotical disputes about the von Neumann probe are only concerned with another isomorphic representation of the same problem of syntax-semantics distinction?

If we refute this due to rejecting the reduction of semiotics to metamathematics, then with Pattee we enter the semiotics, where "(...) even the simplest system requires a code and an interpreter to introduce objective function or meaning to a physical system, and such a system has no obvious precursors" [2, p. 282]. As a necessary part of semiotics, we see the necessity of the process of interpretation and we will thus subscribe to the tradition of semiotics as articulated by Peirce. As a negative consequence, we will remain with theory/theories lacking simplicity and conceptual clarity in the case of definition of the simplest semiotic system. With regard to biosemiotics, we will still be in a situation where its position as a discipline is not clarified (still rather reminiscent of philosophy) and may still be assailed by opponents from the domain of science.

\section{Towards a Comprehensive Description in Life Sciences}

Endorsing Peircean (bio)semiotic tradition primarily implies considering concepts such as interpretation, agent and contextual dependency as unavoidable. Our argumentation could therefore be built on various levels around the above-cited system constraints, the characteristics of complementary modes as the essential existential condition of living systems and finally by taking different conceptions of the epistemic cut into account.

We return here to various constraints described by Kull and Emmeche [8, p. 219]. As averred above, it is possible to discuss the relevance of particular levels for biosemiotics. Radical acceptance of descriptiveconstructive differentiation as the sole relevant constraint contrasts sharply with the contextual dependency of meaning construction $[9,10]$

10 In connection with our metamathematical consideration of the basic semiotic situation, we should remember that in all three theories above (which mirror the same conceptual necessity of distinction between syntax and semantics) is present a condition known from the set theory namely, the impossibility of realizing unambiguous pairing between an infinite set of natural numbers and an infinite set of real numbers. Simply put, the basic common property behind the mirroring of true sentences, acceptable strings and representable codes is based on the impossibility of ordering real numbers. That is why we have an infinite number of true but incomprehensible sentences, acceptable,but undecidable strings and representable but unbuildable codes. 
Let us now consider these physical constraints of non-living local structure types [11, p. 315] and their relevance for development and function of the particular sign. Here, one can readily see that the functional potential of a sign vehicle depends on its physical constitution, which in turn is directly connected to physical conditions and thus to constraints imposed by the characteristics of natural surroundings. ${ }^{11}$

The contextual dependency could thus be documented by principles of ontogenesis. As an example, we can cite differing phenotypical manifestations of monozygotic twins or simply any possible physical-chemical effect on random organism. More specifically, we cite the effect of teratogens on foetal development or other gene mutations that can occur during an individual's life. These effects can therefore be regarded as contextual conditions for a specific form of life. ${ }^{12}$

\section{Complementary Modes: Conditions for the Existence of Life}

A contextual dependency of the meaning, the necessary existence of an agent as a subject of semiotic activity and interpretational potential of the autonomous system itself, is now reflected through a description of two complementary modes and their conception in a discourse of biosemiotics. We elaborate one of Pattee's fundamental theses, namely, a postulate that all living systems are dependent on the interaction between two types of complementary modes [12,13]. According to Pattee, these complementary modes differ mainly in relation to (1) their time coordinates and (2) their internal characteristics - whether they are discrete or continuous. Thus, we can distinguish a time-independent, or linguistic, mode that disposes of a potential of symbolic record and a dynamic mode characterized by its time dependency.

Reflecting on Pattee's differentiation, it is clear that these complementary modes are essentially identical or analogous to other oppositions, especially to duality of analogue and digital codes (or code for action and the code for memory) as depicted by Hoffmeyer (e.g. 2008).

In the context of the main properties of digital codes, Hoffmeyer [18, pp. 80-92] emphasizes three of them that reflect the main differences between digital and analogue coding. The first is that messages expressed in digital codes do not have to observe the limitations of freedom imposed by natural laws. Therefore, we are able to express impossible as well as possible messages and thus tell stories written even in a distant galaxy ages ago. Hoffmeyer speaks about the second advantage, claiming that digital codes are ideal codes for memory and cites in fact two of those that are related through the very existence of memory itself and match completely those time-independent modes of Pattee (as cited above). These are time independence and consensual objectivity. One the possibilities to emphasize the importance of the former is also reflected by the so-called epochs of communication described e.g. by McLuhan and De Fleur [19, pp. 33-43]. In the context of these characteristics, we think of memory substitution in the sense of written and unwritten records, which features transmission of socially and biologically definable cultures in time. Finally, we can depict this memory substitution in relation to the agent as a unit of a living cultural community. It is hard to imagine how certain types of complex information would have survived intact through time if they had been coded in other rather than in digital code. The digital codes' objectivity depends on a shared convention and is therefore determined by intersubjective relations in a community of various agents. The third advantage of digital codes is that they serve as tools for abstraction. It is important for metacommunication, which significantly depends on digital coding, although it is not the only way to construct simple metamessages. Advanced abstractions as processes presume the existence of the autonomous agent possessing a certain type of an action potential.

In depicting the digital code, we covered its main properties. However, speaking of code duality, the requirement of complete description arises on both sides. Therefore, it is necessary to add some other essential features to the concept of analogue code. Argumentation for acceptance of descriptive extension in life sciences thus needs to be supplemented by analogue coding features that are not implied by the features and the differentiations of above digital coding.

11 In the field of semiotics, the degree of the form on function dependency can vary. Within social behavior conventional rules that assign certain functions for particular object undoubtedly confirm this statement. peaking of the semiotic control of autonomous systems and their environment, the form on function dependency seems inescapable.

12 Within the scientific literature, the concept of context is commonly used with starkly different meanings. Aware of this, we use the term in the general sense of related phenomena and events. Interfaces of these types themselves could then be considered as the specific objects of interest in several disciplines. 
The main aspect of the analogue code is meaning complexity. By using the digital domain of signification, we can only draw near the rich semantics of analogue form. As the semantics is one of the main distinctions, the level of syntax is the very next. The analogue form does not possess the rich syntax of the digital domain [18, pp. 82-92].

However, the boundaries emerging from the description are blurred. Therefore, the differentiation should rather be built on particular characteristics, not on their complete concurrent validity. This conception thus corresponds to one of Bateson's key statements: "In the natural world, communication is rarely either purely digital or purely analogic" [20, p. 291]. In relation to Peircean (bio)semiotics of meaning (or function), it is then necessary to refer to a concept of Gestalt shift (or switch) when a type of coding depends on the context and interpretation. For a particular form or constitution, the variation between digital and analogue coding could thus be detected.

\section{The Epistemic Cut and the Agent of Semiosis}

The complementarity principles of discrete (symbolic) and continuous (dynamic) modes are keystones for a concept of a broader meaning. Since Newton's separation of laws and initial conditions, it is possible to observe the necessity of certain conceptual and also significantly arbitrary differentiation of subject and object. The significance of this step is also emphasized in the work of physicists including Heisenberg, Schrödinger, Pauli, von Neumann and Wigner. It is a physical and logical requirement to make sense of any measurement, record, observation or empirically testable theory [21, p. 9]. This differentiation (further applied on concepts) of symbol and matter is the epistemic cut.

Traditional philosophical approaches usually deal with principles of epistemic cut on a cognitive level. The essence of these approaches thus lies in issues of reference and characteristics of processes where symbols become entities that control matter and acquire meaning [22]. However, for Pattee, the essence of the concept is a more fundamental question. He rather focuses on the emergence of meaning and thus on the question of the emergence of life itself: "How do material structures ever come to be symbolic?" [21, p. 230].

In light of the above, a key understanding emerges from von Neumann [6] who refers to the epistemic cut as a separation of the measuring device from what is measured. Principles of this separation are then analogous in relation to description-construction differentiation. Pattee extends these analogies and defines the epistemic cut as a separation of the observer from the observed or between the symbolic record of an event and the event itself $[21$, p. 8].

One of the major inadequacies for the concept of the epistemic cut is often an imperfect or rather inconsistent analogy of antithetical concepts. However, a number of commonly accepted assumptions demonstrate the need to take the autonomous agent into account when talking about semiotic control of system itself. As a broad illustration, let us first point out those incommensurable pairs that are ordinarily used as fundamental elements of the concept: (1) subject vs. object, (2) observer vs. (what is) observed and (3) symbolic record vs. event.

By briefly evaluating these analogies, we could reveal the variance in characteristics of subject-object against symbolic record-event (itself) opposition. The symbolic record thus also could, or sometimes must, switch to become perceived as the object or what is observed. For us, it is also worth noting that essential features of concepts obviously imply the existence of the somehow active agent as the entity performing the meaningful activity, e.g. any kind of operations in relation to the record.

However, the agent's role is a vague concept because it is also closely linked to a wide range of conceptualizations of the epistemic cut itself. Thus, we can observe the understanding of agent's concept in the sense of observer who defines or decides what is observed and determines where to apply the epistemic cut. The essence of epistemology is thereby reflected as well as the tendencies to dealing with the concept on a cognitive level.

Early interpretations of quantum theory led to the labelling of the agent's consciousness as the ultimate epistemic cut that terminates an endless regress to larger and larger coherent quantum systems. Pattee then proposed the equivalence of quantum measurement and processes of functional behaviour. Fundamental realization of epistemic cut is thus closely linked to the origin of life. The notion of the simplest agent as an entity possessing the ability to (re)act given by the semantic closure could then be considered as a satisfactory definition [23, 24, pp. 9-28]. According to Pattee, the cell might therefore be conceived as the simplest (form of) agent $[21$, p. 9]. 


\section{Conclusion}

For a discipline concerned with natural and live phenomena, it is surely indispensable to insist on using clearly defined tools of explanation. This type of explanatory potential is reflected by the assignation of the reliable code existence as the primary and sole condition for semiosis and therefore as the very existence of self-regulating living systems. A theoretical basis concerning this argumentation and its advantages for description thus formed the first part of this paper.

In the second part, we extended the argumentation by means of generally accepted approaches in (bio)semiotics. We proceeded with a description and consistent analysis by means of terms such as context, interpretation or the autonomous agent.

The major question remaining is how much of the ability to describe and explain the questions of life we would lose (or not) by rejecting the explanatory extensions mirrored in Peircean tradition. However, to establish biosemiotics as a firm science, it is advisable to keep in mind principles of reductive and rigorous explanations before accepting these explanatory extensions.

We do not advocate that readers choose sides. The dialogic nature of this paper strove to point out that these approaches are not mutually exclusive (and are even seen as mutually supportive). All these arguments considered, let us quote Kull and Emmeche [8] with probably the most appropriate conclusion for the paradigm of the life sciences themselves: "our biosemiotic models require many different levels of descriptions. Failure to recognize that these different levels of descriptions are necessary and complementary often causes useless arguments over which is the 'best' description".

\section{Acknowledgements}

This work was supported by the student project "Living Systems in Contexts at Faculty of Arts of the Palacky University"(IGA_FF_2018_020).

\section{References}

[2] Pattee. H. H., 2005. The Physics and Metaphysics of Biosemiotics. Journal of Biosemiotics, 1, pp. 281301.

[3] Batterman R., 2013. The Oxford Handbook of Philosophy of Physics. Oxford University Press.

[4] Faltýnek, D., Jastřembská, K., 2014. Součet s nulou: všem, kteři nenásobí nulou. Olomouc: Univerzita Palackého v Olomouci, Qfwfq.

[5] Barbieri, M., 2015. Code Biology: A New Science of Life. New York: Springer.

[6] von Neumann, J., 1966. Theory of Self-reproducing Automata. London: University of Illinois Press.

[7] Partee, B., Barbara B.H., ter Meulen, A.G., Wall, R., 1993. Mathematical Methods in Linguistics. London: Kluwer Academic Publishers.

[8] Kull, K., Emmeche, C., c2011. Towards a Semiotic Biology: Life is the Action of Signs. Hackensack, NJ: Distributed by World Scientific.

[9] Markoš, A., 2010. Jazyková metafora živého. Červený Kostelec: Pavel Mervart.

[10] Švorcová, J., 2007. Organické kódy a paměti. Diploma Thesis, Charles University in Prague.

[11] Kull, K., Pattee, H. H., 2009. A Biosemiotic
Conversation: Between Physics and Semiotics. Sign Systems Studies, 37.

[12] Pattee H. H., 1972. Physical problems of decision making constraints. International Journal of Neuroscience, 3 , 99

[17] Hoffmeyer, J., Emmeche, C., 2008. A legacy for living systems: Gregory Bateson as precursor to biosemiotics. New York: Springer. Biosemiotics 2

[18] Hoffmeyer, J., 2008. Biosemiotics: An Examination into the Signs of Life and the Life of Signs. Scranton: University of Scranton Press, Approaches to Postmodernity, v. 2.

[19] McQuail, D., 2002. Úvod do teorie masové komunikace. Praha: Portál

[20] Bateson, G., 1972. Steps to an ecology of mind: collected essays in anthropology, psychiatry, evolution, and epistemology. Northvale, N.J.: Aronson

[21] Pattee, H. H., Rączaszek-Leonardi, J., c2012. Laws, Language and Life: Howard Pattee's Classic papers on the Physics of Symbols with Contemporary Commentary. New York: Springer. Biosemiotics 7. 
[22] Harnad, S., 1990. The Symbol Grounding Problem. Physica D, 42, 335-346.

[23] Sharov, A., 1998. Signs and values. Virginia Polytechnic Institute and State University, Blacksburg. Available from: http://alexei.nfshost. $\mathrm{com} / \mathrm{biosem} / \mathrm{txt} / \mathrm{isas} 98 . \mathrm{html}$

[24] Pattee, H. H., 1995. Evolving Self-Reference: Matter, Symbols, and Semantic Closure. Communication and Cognition - Artificial Intelligence 12, 9-28. 